clinical impact on morbidity and mortality of patients with chronic HF (CHF) remains to be determined.

Purpose To evaluate the prevalence, clinical associations and prognostic value of three different malnutrition indices in a large cohort of patients referred to a community HF clinic with suspected HF.

Methods Consecutive referrals were analysed. HF was defined as signs or symptoms and evidence of cardiac dysfunction, either a reduced left ventricular ejection fraction at echocardiography (LVEF, <50\%) or raised NTproBNP (>125 ng/L). Patients were screened for malnutrition using three common malnutrition indices: the geriatric nutritional risk index (GNRI), the controlling nutritional status (CONUT) score and the prognostic nutritional index (PNI).

Results Of the 5012 patients who attended between 2000 and 2016, HF was confirmed in 3386 (61\% males, median age 75 (interquartile range (IQR): 67-81) years, median NTproBNP 1573 (IQR: 702-2799) ng/L). Of these, 1198 (35\%) and 1458 (43\%) patients had HF with reduced (HeFREF, LVEF<40\%) and normal (HeFNEF, LVEF 50\%) ejection fraction, respectively. According to the GNRI (91\%), CONUT score (>4) and PNI (38\%), 6.7\%, 10.0\% and 7.5\% patients were moderately to severely malnourished, respectively.

Compared to those with normal nutritional status, malnourished patients were older, had lower body mass index (BMI), worse symptoms and renal function; they were also more likely to have atrial fibrillation, anaemia and reduced mobility.

During a median follow-up of 1573 days (interquartile range: 702-2799 days), 1723 (50.9\%) patients died. In multivariable models, indices of malnutrition were powerful predictors of mortality. In a multivariable model that included all the malnutrition indices, only GNRI was independently associated with increased risk of mortality (Hazard ratio (95\% confidence interval): 1.25 (1.12-1.39), $\mathrm{p}<0.001$ for worsening malnutrition category of GNRI).

Conclusion Moderate or severe malnutrition is highly prevalent amongst patients with $\mathrm{CHF}$ and is strongly related to mortality.

\section{IMPLANTABLE CARDIAC DEVICE GENERATOR CHANGE PATHWAYS: AN OPPORTUNITY FOR OPTIMISING DEVICE PRESCRIPTION}

${ }^{1}$ Helena Bolam*, ${ }^{2}$ Daniel Thompson, ${ }^{2}$ James Waller, ${ }^{2}$ Neil Andrews, ${ }^{2}$ Senthil Kirubakaran, ${ }^{2}$ Paul Kalra, ${ }^{2}$ Geraint Morton. ' Queen Alexandra Hospital; ${ }^{2}$ QA Hospital

\subsection{6/heartjnl-2017-311726.7}

Introduction Implantation of cardiac devices including permanent pacemakers (PPM), cardiac resynchronisation therapy with defibrillators (CRT-D) or without (CRT-P) is increasingly common. Follow up, including arranging generator change, is frequently carried out by cardiac physiologists, with minimal physician input. Many patients clinical status will change during the lifetime of the device. Generator change is an opportunity to review the original device prescription. We report the impact of introducing routine screening prior to generator change into an established comprehensive device programme. Methods Two pathways were implemented. Patients with a PPM due generator change were screened by cardiac physiologists using a proforma assessing symptoms, QRS duration and right ventricular (RV) pacing burden. Those with heart failure symptoms, in particular breathlessness, and a prolonged native
QRS or $>40 \%$ RV pacing had an echocardiogram and multidisciplinary team (MDT) discussion within 2 weeks. If device upgrade was potentially indicated the patient was reviewed in clinic prior to generator change.

The goal was to discuss all patients with an ICD/CRT-D due generator change at MDT. Those with changes in clinical status that may affect the appropriateness of ongoing defibrillator therapy were reviewed in clinic.

Results The first 11 months, October 2015 to August 2016, following implementation of these pathways were studied. 189 consecutive patients (145 PPM, 20 CRT-P and 24 CRT-D/ ICD), mean age of 77 , were included.

The pathways resulted in a change in the type of device therapy in 8 patients; 6/145 (4\%) of those due PPM and 2/ $24(8 \%)$ due CRT-D/ICD generator change (table 1).

$145(100 \%)$ of PPM patients were proforma screened. 139/ 145 PPM patients (96\%) went on to have PPM generator change. 17 (12\%), identified as potential candidates for upgrade to CRT, had echocardiography. 5 (3\%) of these had severe LV impairment and underwent physician and MDT review. 4 patients (3\%) were ultimately upgraded to CRT devices. CRT was not appropriate in 1 patient. In $2(1 \%)$ patients ongoing device therapy of any kind was felt to be inappropriate (1 potential upgrade and 1 PPM box change) and no procedure was undertaken.

$10 / 24(42 \%)$ patients due a CRT-D/ICD generator change were reviewed at MDT. Of these 2 (20\% of those reviewed) went on to have their devices downgraded from CRT-D to CRT-P after informed discussion.

Conclusions Physiologist-led device follow up can be effectively extended to include robust screening of patients to ensure they get the most appropriate device at the time of generator change. Excellent rates of PPM screening were achieved whereas ICD screening needs to be improved.

Relatively few patients had their destination device changed as a result of these pathways. However there is significant morbidity associated with receiving inappropriate device therapy, justifying implementation of a simple screening process to improve care.

Abstract 7 Table 1 Outcomes for patients due implantable cardiac device generator change after the implementation of routine screening

\begin{tabular}{ll}
\hline Outcomes & Number of patients \\
\hline Type of device unchanged & 181 \\
Upgrade from PPM to CRT-P & 2 \\
Upgrade from PPM to CRT-D & 2 \\
Downgrade from CRT-D to CRT-P & 2 \\
Device therapy withdrawn & 2 \\
\hline
\end{tabular}

\section{THE HF-CGM STUDY: AN ANALYSIS OF CARDIOGONIOMETRIC AXES IN PATIENTSWITH CARDIAC RESYNCHRONISATION THERAPY}

${ }^{1}$ Oliver I Brown*, ${ }^{2}$ Theodora Nikolaidou, ${ }^{2}$ Gowan Beddoes, ${ }^{2}$ Angela Hoye, ${ }^{2}$ Andrew L Clark. ${ }^{1}$ Hull York Medical School; ${ }^{2}$ Hull and East Yorkshire Hospital NHS trust

\subsection{6/heartjnl-2017-311726.8}

Introduction The HF-CGM study is a proof-of-principle study to investigate whether cardiogoniometry (CGM), a three- 